\title{
ASSISTÊNCIA DE ENFERMAGEM AO PACIENTE COM ESTOMIA INTESTINAL: UMA REVISÃO BIBLIOGRÁFICA
}

\author{
NURSING CARE FOR INTESTINAL STOMY PATIENTS: A \\ BIBLIOGRAPHIC REVIEW
}

Karla Pereira Vasconcelos ${ }^{1}$ Cleisla Tamires Lacerda Silva ${ }^{2}$

\begin{abstract}
RESUMO: OBJETIVOS: identificar e descrever os cuidados de enfermagem ao paciente com estomia intestinal. MÉTODOS: Trata-se de uma revisão na literatura de artigos publicados a partir de 2015 a 2019, nas bases de dados Lilacs, Pubmed, SciELO e Google Acadêmico. RESULTADOS: Foram selecionados 9 artigos que estavam dentro da temática abordada e dos critérios de inclusão para realização deste estudo. Foi observado que a assistência de enfermagem, na grande maioria dos casos eram restritas a questões fisiológicas, como o uso correto do dispositivo coletor. Percebeu-se ainda, deficiência em orientações relacionadas ao autocuidado, apoio emocional, psicológico e sexualidade, além de déficit em questões relacionadas a dispositivos que garantem os direitos legais dos estomizados. CONCLUSÃO: A assistência de enfermagem para pacientes estomizados deve ser planejada de modo a complementar seus aspectos fisiológico, psicológico, emocionais, sexuais e sociais, na perspectiva de prestar uma assistência de forma holística. Orientações fragmentadas e pautadas apenas em questões fisiológicas demostram uma formação fortemente pautada no modelo biomédico e carece de ser trabalhada na acadêmia, para que assim seja prestada assistência de qualidade. Desse modo, deve haver uma melhora do processo educativo dos enfermeiros aos pacientes estomizados, de forma que englobe o paciente de modo integral, além de ser crucial a discurssão no meio acadêmico a respeito da assistência de enfermagem ao paciente ostomizado, sendo um tema que carece de mais pesquisas e maior socialização durante a formação dos profissionais de enfermagem.
\end{abstract}

Palavras chave: assistencia de enfermagem; estomia; pacientes.

\footnotetext{
1 Graduanda do Curso de Enfermagem da Universidade Estadual da Paraíba - UEPB, karlapv2017@gmail.com.

2 Graduanda do Curso de Enfermagem da Universidade Estadual da Paraíba - UEPB, cleislalacerda@gmail.com.
} 
ABSTRACT: OBJECTIVE: to identify and describe nursing care for patients with intestinal ostomy. METHODOLOGY: This is a literature review of articles published from 2015 to 2019, in the databases Lilacs, Pubmed, SciELO and Google Scholar. RESULTS: Nine articles were selected that were within the theme and inclusion criteria for this study. It was observed that nursing care in the vast majority of cases were restricted to physiological issues, such as the correct use of the collecting device. There was also a deficiency in self-care guidelines, emotional, psychological and sexual support, as well as a deficit in matters related to devices that guarantee the legal rights of ostomized patients. CONCLUSION: Nursing care for ostomized patients should be planned to complement their physiological, psychological, emotional, sexual and social aspects, with a view to providing holistic care. Fragmented orientations based only on physiological issues show a formation strongly based on the biomedical model and need to be worked in the academy, so that quality assistance is provided. Thus, there should be an improvement in the educational process of nurses to ostomized patients, so that it encompasses the patient in its entirety, and it is crucial to discuss in the academic environment about nursing care for ostomized patients, being a theme that needs more research and socialization during the training of nursing professionals.

Keywords: Nursing care, ostomy, patients. 


\section{INTRODUÇÃO}

As Portarias GM 793 de 24 de abril de 2012 e GM 835 de 25 de abril de 2012, que tratam sobre 0 instrutivo ostomia no Brasil, definem estomia como o procedimento cirúrgico que compreende na exteriorização do sistema, seja ele digestório, respiratório ou urinário, gerando um orifício externo chamado de estoma, e ostoma com a intervenção cirúrgica que cria o ostoma (abertura) na parede abdominal para colocação da bolsa de fezes ou urina (BRASIL, 2012). A Portaria SAS/MS n 400, de 16 de novembro de 2009, que trata da atenção à saúde de pessoas ostomizadas no Brasil, conceitua estomia intestinal (colostomia e ileostomia) como uma intervenção cirúrgica realizada no intestino grosso (cólon) ou no intestino delgado onde é feita a exteriorização de um segmento intestinal por meio da parede do abdome, desta forma originando uma abertura artificia para a saída de fezes (BRASIL, 2009).

A cada ano ocorrem no mundo cerca de 945 mil novos casos de câncer que acometem o cólon e o reto, sendo o segundo tipo de câncer mais prevalente no mundo e tendo um prognóstico considerado de moderado a bom. Segundo o INCA (Instituto Nacional de Câncer) para o ano de 2018, havia uma estimativa de 36.360. novos casos, acometendo 17.380 homens 18.980 mulheres (INCA, 2018). Esses novos casos, são resultado em grande parte de cirurgias traumatizantes e mutilantes, que provocam alterações significativas no modo de vida das pessoas afetadas (BRASIL, 2019). A estomia é considerada de grande relevância por ser um procedimento cirúrgico que possibilita a sobrevida de pacientes acometida por câncer colorretal (MAURICIO, et al., 2017).

Segundo Rocha (2011), as principais indicações para os estomas intestinais são: obstrução intestinal (agenesias e atrésias anorretais, megacólon congênito, neoplasias, volvo, doença diverticular e colite isquêmica), perfurações do cólon (neoplasias, doença inflamatória intestinal, doença diverticular, colite isquêmica), traumas (penetrante com arma branca ou de fogo, fechado e empalação), fístulas 
(anorretais, reto-vaginais, reto-vesicais), e proteção de anastomoses de alto risco (colorretais, colo-anais e ileo-anais).

A pessoa com estomia poderá passar por dificuldades relacionadas ao tratamento, reabilitação e adaptação ao novo estilo de vida, essa dificuldade por sua vez pode vir a causar conflitos de pensamentos e emoções, e para que estes sejam evitados, é necessário que a assistência seja prestada de modo integral, considerando os diversos aspectos fisiopatológicos, nutricionais, psicológicos, sociais e espirituais da pessoa ostomizada. Para tanto, deve-se considerar as características individuais, o contexto familiar, cultural, religioso, comunitário, social, econômico, de escolaridade, dentre outros (Silva et. al, 2017).

As principias alterações demostradas na vida dos pacientes submetidos a procedimentos de ostomia estão relacionadas a questões como: vida social, imagem corporal, perda do controle sobre o corpo e alterações nas emoções. E foram observados que entre as principais reações e sentimentos causados pela ostomia, destacam-se: vergonha, medo diante da nova situação, constrangimento, malestar com relacionado ao odor, limitação e discriminação. (SOUZA, et al., 2016). Assim, em se tratando de reações e sentimentos, pode-se verificar que é necessário investir no contexto emocional junto ao paciente ostomizado para que seja possível a reconstrução de sua autoimagem, sendo necessário apoia-lo a viver com sua nova imagem corpórea, bem como, elaborar o luto do corpo da antiga imagem corporal (SOUZA, et al., 2016).

A estomia pode ser utilizada de forma provisória ou definitiva, porém de toda forma trará impactos na vida do indivíduo devido a perda da continência, emissão de odores desagradáveis, barulho de gases, necessidade de utilizar um equipamento coletor e desta forma mudança no vestuário, entre outras (NASCENTES, 2015).

Existem diversos tipos de ostomias, mas todos os casos carecem de atenção e orientações adequadas a fim de evitar e complicações referentes ao manuseio inadequado de sua ostomia, sendo a participação do enfermeiro neste processo de extrema importância buscando orientar e contribuir com o atendimento adequado e promover aos usuários um cuidado livre de riscos e orientado à promoção da sua saúde (SOUZA, et al., 2016). 
Assim, a estomia é um desafio para a maioria das pessoas, e esses indivíduos carecem de cuidado e atenção qualificada dos profissionais de saúde, visando suprir a demanda de assistência e a educação em saúde, principalmente no que diz respeito ao autocuidado (BRASIL, 2009). A alteração da autoimagem corporal pode refletir negativamente na autoestima, no autoconceito, na sexualidade e, consequentemente, na identidade pessoal do paciente (BARBURITTI, et al., 2008).

Portanto, é importante que não apenas o ostomizado, como também a família esteja envolvida no cuidado à pessoa com estomia. É dever dos profissionais de saúde favorecer a inclusão tanto do indivíduo quanto dos familiares na recuperação e a reabilitação destas pessoas. Nesse contexto, é necessário que o enfermeiro trabalhe junto ao paciente, visando à educação não apenas sobre a ostomia, mas maneira como o paciente imagina a vida com a bolsa coletora, permitindo que 0 paciente expresse seus sentimentos, medos e angústias, e assim seja possível para o enfermeiro ter subsídios necessários para aplicar possíveis intervenções (SOUZA, et al., 2016). Cabe destacar ainda, a importância do papel do enfermeiro, que deve favorecer atividades de autocuidado e segurança do paciente como fator primordial na sua recuperação, bem como intensificar as atividades de educação em saúde trabalho junto à população, visando a melhoria da qualidade de vida, promoção de práticas saudáveis e diminuição dos agravos a sua saúde (SOUZA, et al., 2016).

A assistência para esses pacientes deve ser planejada de modo a complementar seus aspectos fisiológico e o psicológico, na perspectiva de prestar uma assistência integral. Para tanto, é importante que uma equipe multiprofissional participe de modo efetivo do processo de atendimento, caracterizado como seguimento contínuo toda a duração da hospitalização, o tratamento cirúrgico, bem como o cuidado continuado (BRASIL, 2019). Deve-se, planejar intervenções psicológicas pré e pós-operatórias, visando desde o preparo para a confecção do estoma até a adaptação ao novo estilo de vida. Essas estratégias, possibilitam conhecer as percepções individuais sobre a doença e o adoecimento, em vez de centrar exclusivamente sobre as estratégias de enfrentamento que os pacientes utilizam após o procedimento cirúrgico e a confecção da ostomia (BRASIL, 2019). 
Sentimentos negativos, como ansiedade, depressão e angústia, surgem concomitantemente às preocupações sobre a vida social e a insegurança por reintegração depapéis e funções sociais prévios. Dessa forma, os profissionais de saúde devem reconhecer e auxiliar/estimular os pacientes em seus esforços para diminuir essas preocupações oferecendo suporte profissional adequado.Primordialmente, deverá ser estimulada a capacidade de realizar os cuidados com a estomia e pele periestoma, a competência para identificar problemas e complicações, além de buscar soluções apropriadas, de cunho físico e psicossocial (BRASIL, 2019).

Como técnica utilizada para o enfrentamento, existe a prática de interação grupal entre pacientes que vivem a mesma situação, que é recomendada em vários estudos e mostra a importância de um acompanhamento especializado após a alta hospitalar, utilizando acolhimento e autocuidado para ajudar o paciente à superar os problemas e retomar sua vida (BRASIL, 2019).

Desta forma, este estudo tem como objetivo identificar e descrever os principais cuidados de enfermagem empregados na assistência ao paciente com estomia intestinal.

\section{METODOLOGIA}

Trata-se de uma pesquisa realizada através de uma revisão na literatura sobre a assistência de enfermagem aos pacientes colostomizados. O levantamento bibliográfico foi realizado no mês de novembro de 2019, utilizando as bases de dados: Literatura Latino- americana e do Caribe em Ciências da Saúde (Lilacs), United States National Library of Medicine (Pumed), Scientific Eletronic Library Online (SciELO) e Google Acadêmico, usando-se como descritores: "assistência", "enfermagem", "colostomia" e "ostomia".

Utilizou-se como critérios de inclusão trabalhos completos disponíveis nas plataformas de pesquisa, com publicação em português entre 2015 e 2019, e que abordassem a assistência de enfermagem aos pacientes colostomizados. Foram 
excluídos anais de congresso e teses, ou que não citassem o profissional de enfermagem como provedor de cuidados. Foi realizada a leitura dos resumos dos artigos e foram excluídos aqueles que não atendiam ao objetivo do estudo. Após avaliação, foram excluídas 133 publicações, e identificados 9 artigos que preencheram todos os critérios.

\section{RESULTADOS E DISCUSSÃO}

Após leitura dos resumos dos 142 artigos, foram selecionados 34 artigos, que foram lidos integralmente. Depois de serem analisados, 9 artigos foram selecionados como objetivo deste estudo, pois estavam em conformidade com o objetivo da pesquisa e com os critérios de inclusão. Os demais foram excluídos devido repetição, não apresentarem texto na íntegra e estarem dentro dos critérios de exclusão.

O quadro 1 foi elaborado para demonstrar quais foram os artigos estudados e seus objetivos, sintetizando de forma clara e organizada os títulos, autores, objetivos e ano de publicação dos artigos.

Quadro 1 - Autores, títulos, anos de publicação e objetivo dos artigos selecionados.

\begin{tabular}{|c|c|c|c|}
\hline Autores & Título & Ano & Objetivo \\
\hline $\begin{array}{l}\text { Coqueiro; } \\
\text { Rodrigues; } \\
\text { Figueiredo }\end{array}$ & $\begin{array}{l}\text { A produção do cuidado } \\
\text { ao usuário estomizado: } \\
\text { considerações da } \\
\text { equipe de enfermagem }\end{array}$ & 2015 & $\begin{array}{l}\text { Conhecer as concepções da equipe } \\
\text { de enfermagem sobre o cuidado ao } \\
\text { usuário estomizado. }\end{array}$ \\
\hline Freire et al. & $\begin{array}{l}\text { Autoimagem } \\
\text { autocuidado na vivência } \\
\text { der pacientes } \\
\text { estomizados: o olhar da } \\
\text { enfermagem }\end{array}$ & 2019 & $\begin{array}{l}\text { Analisar a percepção de pacientes } \\
\text { estomizados sobre a sua } \\
\text { autoimagem e autocuidado. }\end{array}$ \\
\hline Maurício et al. & $\begin{array}{l}\text { A visão dos enfermeiros } \\
\text { sobre as } r \text { práticas } \\
\text { educativas direcionadas } \\
\text { as } \\
\text { estomizadas }\end{array}$ & 2017 & $\begin{array}{l}\text { Analisar o ponto de vista dos } \\
\text { enfermeiros sobre as ações } \\
\text { educativas realizadas com as } \\
\text { pessoas com estomia visando à } \\
\text { inclusão social. }\end{array}$ \\
\hline
\end{tabular}




\begin{tabular}{|c|c|c|c|}
\hline $\begin{array}{l}\text { Mendonça } \\
\text { al. }\end{array}$ & $\begin{array}{lrr}\text { Orientações } & \text { de } \\
\text { enfermagem } & \text { e } \\
\text { implicações para } & \text { a } \\
\text { qualidade de vida de } \\
\text { pessoas estomizadas }\end{array}$ & 2015 & $\begin{array}{l}\text { Analisar as repercussões das } \\
\text { orientações de enfermagem } \\
\text { fornecidas aos clientes estomizados } \\
\text { que frequentam o Grupo de Apoio, } \\
\text { na perspectiva do alcance de uma } \\
\text { melhor qualidade de vida. }\end{array}$ \\
\hline $\begin{array}{l}\text { Moraes; } \\
\text { Santos; Borge }\end{array}$ & $\begin{array}{lr}\text { Da formação à prática: } \\
\text { a percepção } \quad \text { de } \\
\text { supervisores } & \text { de } \\
\text { enfermagem sobre os } \\
\text { cuidados em estomias }\end{array}$ & 2016 & $\begin{array}{l}\text { Descrever a assistência de } \\
\text { enfermeiros } \\
\text { relacionada ao cuidado do paciente } \\
\text { estomizado em ambiente hospitalar. }\end{array}$ \\
\hline $\begin{array}{l}\text { Mota; } \\
\text { Gomes }\end{array}$ & $\begin{array}{l}\text { Vida e sexualidade de } \\
\text { mulheres estomizadas: } \\
\text { subsídios à } \\
\text { enfermagem }\end{array}$ & 2016 & $\begin{array}{l}\text { Conhecer como a estomização } \\
\text { interfere no viver e na sexualidade } \\
\text { de mulheres estomizadas. }\end{array}$ \\
\hline Nieves et al. & $\begin{array}{l}\text { Percepção de pacientes } \\
\text { ostomizados sobre os } \\
\text { cuidados de saúde } \\
\text { recebidos }\end{array}$ & 2017 & $\begin{array}{l}\text { Identificar a percepção de pessoas } \\
\text { com estoma digestivo sobre o } \\
\text { processo de atendimento recebido e } \\
\text { as áreas de melhoria detectadas, } \\
\text { bem como suas necessidades e } \\
\text { sugestões para esse fim. }\end{array}$ \\
\hline Souz & $\begin{array}{l}\text { Apoio emocional } \\
\text { realizado por enfermeiro } \\
\text { ao paciente ostomizado }\end{array}$ & 2016 & $\begin{array}{l}\text { Identificar reações causadas pela } \\
\text { ostomia, medidas adotadas a partir } \\
\text { do desconforto percebido pelo } \\
\text { paciente ostomizado e a importância } \\
\text { do apoio emocional proporcionado } \\
\text { pela enfermagem. }\end{array}$ \\
\hline Vera et al. & $\begin{array}{llr}\text { Sexualidade } & \text { e } \\
\text { qualidade } & \text { de vida da } \\
\text { pessoa } & \text { estomizada: } \\
\text { reflexões para } & \\
\text { cuidado de } & \\
\text { Enfermagem } & \end{array}$ & 2017 & $\begin{array}{l}\text { Refletir sobre a atividade sexual } \\
\text { como dimensão importante na } \\
\text { qualidade de vida e no processo de } \\
\text { cuidar em enfermagem no manejo } \\
\text { de pessoas estomizadas. }\end{array}$ \\
\hline
\end{tabular}

O procedimento de estomização ocasiona mudanças que podem impactar negativamente o indivíduo no lado físico, psicológico, sexual e emocional (VERA et al. 2017). Apesar da bolsa de colostomia ser utilizada para fornecer uma qualidade de vida ao paciente, percebe-se que o mesmo passa por dificuldades de adaptação e de aceitação, baixa autoestima, sentimentos negativos que geram sofrimento, desta forma é muito importante os cuidados da equipe multidisciplinar para acompanhar, orientar, e incentivar sobre a adaptação (FREIRE et al., 2019). 
O aumento do número de pessoas estomizadas no Brasil reforça a importância do profissional de enfermagem no processo de educação em saúde a esta população, utilizado como um instrumento para prestar uma assistência de qualidade, estas orientações proporcionam um suporte psicossocial, melhorando a qualidade de vida do paciente (MAURÍCIO et al., 2017).

Freire et al. (2019), citam em seu estudo que houve uma participante que demonstrou escolher o isolamento social após colocação da bolsa de colostomia, e os autores destacam ainda que esta questão deve ser verificada pelos profissionais de saúde, em especial o enfermeiro, de forma a mudar esse olhar do paciente e melhorar sua adaptação, criando uma rede social de apoio.

As orientações de enfermagem devem ser realizadas de forma holística, observando a recuperação nos lados biopsicossocial do indivíduo. Para a reabilitação do cliente, é necessário o ensino do autocuidado, buscando recuperar a autoestima e incluindo o paciente no meio social. Assim o paciente irá retornar às atividades de vida diária e seus planos, obtendo qualidade de vida (MENDONÇA et al., 2015). Os pacientes estomizados merecem uma atenção especial dos profissionais, serviços e programas de saúde, pois os mesmos possuem dificuldades físicas e psicossociais, e desta forma exigem intervenções especificas direcionadas a reabilitação (VERA et al., 2017).

Desta forma, no processo de educação em saúde, o enfermeiro deve realizar um atendimento individualizado, voltado às particularidades do cliente, e não direcionar apenas para os cuidados com a pele e com a bolsa de colostomia, pois as necessidades dos pacientes estomizados abrangem o lado psicológico e social, e não só o físico (MENDONÇA et al., 2015). O enfermeiro deve oferecer um apoio emocional, mostrando que está disponível para auxiliá- lo, através de demonstração de respeito, afeto, segurança, atenção, ofertando informações, incentivando o paciente. (SOUZA et al., 2016).

É essencial observar o contexto social no qual o cliente se encontra, pois alguns clientes não retornam as consultas com frequência, muitos pacientes são financeiramente carentes, o que dificulta a necessidade de se transportar até as consultas (MAURÍCIO et al., 2017), mostrando a importância da atenção aos 
pacientes colostomizados na atenção básica, não somente na atenção especializada.

Como educador, o enfermeiro deve orientar sobre a saúde e autocuidado, e para isso deve saber do contexto socioeconômico do paciente, de suas condições físicas e psicoemocionais (MENDONÇA ET AL., 2015). O grau de instrução deve ser levado em consideração no processo de educação, devido a capacidade de compreensão do paciente e para o desenvolvimento de habilidades que levem a reabilitação (MORAES; SANTOS; BORGES, 2016).

O enfermeiro também deve fornecer orientações sobre os direitos legais dos estomizados, indo desde os aspectos técnicos a seus direitos como cidadãos. Essas informações melhoram a qualidade de vida dos indivíduos, pois proporcionam uma economia financeira e maior acesso aos serviços de assistência social e de saúde (MENDONÇA ET AL., 2015).

Freire et al. (2019) identificaram em seu estudo que o uso da colostomia ocasiona sentimentos de vergonha, insegurança, invasão e sofrimento, além do paciente demonstrar dificuldades de adaptação e aceitação que levam ao isolamento social, pois sentem medo de exclusão, gerando alterações psicológicas, emocionais e sociais. Trazem ainda que suas expectativas são relacionadas a necessidade de receber orientações que melhoram sua adaptação, se adequem a sua realidade e os preparem para os obstáculos e complicações que enfrentarão no cotidiano.

O incentivo do enfermeiro a atividade de lazer é muito importante para o paciente retomar sua vida, levando o mesmo a uma melhora da autoestima, frequentar novos ambientes e conhecer pessoas novas, ter divertimento e sentimentos novos, melhorando sua qualidade de vida. A promoção de reabilitação do indivíduo vai se dar se diversas formas, pois deve se adequar a especificidade do indivíduo (MENDONÇA et al., 2015).

Souza et al. (2016) ressaltam que o desconforto que os pacientes estomizados sentem geralmente está relacionado a ausência de orientação sobre o uso da bolsa coletora e sobre o autocuidado, além da falta de apoio emocional, que dificultam uma boa adaptação do paciente. Desta maneira, o paciente deve receber 
apoio emocional dos amigos, familiares, e principalmente do enfermeiro prestador dos cuidados.

Maurício et al. (2017) citam que a maioria dos enfermeiros que faziam parte de seu estudo consideravam bastante relevante o ensino do autocuidado para os pacientes colostomizados, para que desta forma eles possam atingir a autonomia e independência. Oferecer orientações, especialmente sobre o autocuidado, para que os pacientes possam se adaptar à nova condição e retornarem as atividades do dia a dia, inclusive a sexualidade, são fundamentais. (VERA et al., 2017).

O enfermeiro deve ofertar informações adequadas, e orientar sobre onde eles podem conseguir a bolsa coletora, como lidar com os odores e com as mudanças que ocorrerão. Para isso, pode utilizar de vários recursos, como por exemplo, mostrar as bolsas e oferecer materiais informativos, para ajudar na adaptação (SOUZA et al. 2016).

No estudo de Maurício et al. (2017), os enfermeiros entrevistados destacam a importância de orientações nas consultas sobre a condição de saúde do indivíduo, o manuseio, troca e esvaziamento da bolsa de colostomia, que são fundamentais ao processo de adaptação do indivíduo à nova condição de saúde. Porém as autoras destacam que observaram as orientações eram voltadas mais para a questão física, sendo pouco ofertadas orientações psicossociais, mostrando um déficit no processo educativo desses pacientes, dificultando sua reabilitação que necessita de orientações holísticas. Esta importância dada por alguns enfermeiros a questão fisiológica mostram uma formação profissional fortemente influenciada pelo modelo biomédico, de forma que o indivíduo é visto de forma fragmentada, com cuidados voltados ao estoma, uso da bolsa de colostomia e cuidados com a pele, não observando o indivíduo como um todo e sem contribuir para suas dificuldades psicossociais.

Moraes, Santos e Borges (2016), a partir de seu estudo, relatam que o enfermeiro hospitalar não possui conhecimento e práticas suficientes para um atendimento adequado ao paciente estomizado, mostrando o déficit na formação do enfermeiro generalista. Os enfermeiros deste setor ensinam sobre a higiene e troca da bolsa de colostomia, mas não orientam sobre o autocuidado e complicações do estoma, e não referenciam para o serviço especializado. 
Oferecer apoio emocional também se constitui como uma ação profissional, onde o paciente irá receber amparo, segurança, orientações, suporte e proteção. Para ofertá-lo, é necessário que o enfermeiro tenha conhecimentos sobre o assunto, se envolva com o paciente e ofereça escuta adequada, além de possuir habilidade técnica e interpessoal, e sensibilidade para identificar as necessidades do cliente e de seus familiares. Também é necessário que o enfermeiro respeite o tempo necessário para o paciente compreender e se adaptar à nova condição, de forma que se mostre a disposição, mostrar que o paciente não está só e que pode conviver com a estomia (SOUZA et al. 2016).

Em pesquisa com pacientes ostomizados, Nieves et al. (2017) relataram que os mesmos identificam as funções do enfermeiro estomaterapeuta, com destaque ao fornecimento de informações e treinamento para que eles tivessem uma melhor adaptação. Os pacientes ainda enfatizaram o valor de terem alguém para solucionar dúvidas e preocupações que apresentam, e o incentivo a continuarem no processo. Relatam também que os enfermeiros os ensinam os cuidados do estoma, a escolher o tipo de bolsa, dieta e informar o que há de novidade na área. Também são ensinados sobre a irrigação e como tratar os problemas resultantes do estoma e pele periestomal.

Coqueiro, Rodrigues e Figueiredo (2015) salientam em seu estudo que nos cuidados ao estomizado deve haver uma harmonia entre pele, estoma, peristoma e bolsa coletora, para assim favorecer o autocuidado do paciente e desta forma proporcionar uma melhor qualidade de vida. Para isso, é necessário que o profissional de enfermagem tenha conhecimento sobre as bolsas de colostomia para orientar seu uso adequadamente. Porém, em seu estudo foi constatado que os profissionais não possuem conhecimento suficiente sobre o assunto, de forma que alguns utilizam até mesmo sacos plásticos para coletar os resíduos intestinais, que por serem de baixa qualidade e precisarem ser trocados constantemente, podem causar dermatites irritativas e alergias em volta do estoma, desta forma gerando desconforto e redução na qualidade de vida. Os enfermeiros e técnicos de enfermagem também demonstraram pouco conhecimento sobre as complicações do estoma, uso de adjuvantes para proteção da pele, dispositivo utilizados para 
drenagem e orientações adequadas para os pacientes. Assim, os profissionais oferecem uma assistência fragmentada, que não supri as necessidades do cliente.

Os pacientes do estudo de Nieves et al. (2017) citam o enfermeiro estomaterapeuta como o especialista no cuidado com o estoma, e destacam esses profissionais como essenciais no enfrentamento da situação, devido os cuidados e informações recebidas. Relatam que estes profissionais influenciaram na forma de lidar com as alterações após o estoma, ajudando a superar sentimentos de incerteza e desinformação, levando ao retorno de vida normal. Também destacaram a importância de poder contar com alguém para lhes acompanhar até se sentirem autossuficientes.

Deve haver uma melhora do processo educativo dos enfermeiros aos pacientes estomizados, de forma que englobe o psicossocial, abordando o retorno ao trabalho, a sexualidade, atividades de esporte e de lazer, vestuário, entre outros (MAURíCIO et al., 2017). Mendonça et al. (2015) destaca a importância de pesquisas e socialização das orientações de enfermagem ao paciente estomizado, de forma que é um tema pouco discutido no meio acadêmico e na assistência de enfermagem. As autoras citam ainda que a educação em saúde e as orientações de enfermagem são um cuidado muito importante, pois faz com que a pessoa entenda melhor seu processo de adoecimento, os procedimentos realizados e tenham maior autonomia.

Mendonça et al. (2015) destacam que os pacientes colostomizados entrevistados compartilhavam as orientações recebidas pela equipe de enfermagem com outras pessoas, de forma a também melhorar a qualidade de vida de outros estomizados.

O estudo de Maurício et al. (2017) traz ainda que os enfermeiros entrevistados possuem algumas percepções interessantes, devido suas experiências, sobre o processo educativo: para implantá-lo é necessário uma avaliação inicial dos conhecimentos prévios do paciente; a primeira consulta deve ser rápida, pois os pacientes não assimilam de forma satisfatória o que é ensinado; alguns necessitam inclusive de inicialmente uma atenção psicológica, com escuta ativa, de apoio emocional na primeira consulta. Os profissionais também destacaram que o processo educativo é dinâmico e transformador, de forma que o enfermeiro também 
aprende com os pacientes através da troca de experiências, demonstrando como deve ser o processo educativo, com um intercâmbio de conhecimentos em que todos os sujeitos possuem algum saber e sempre há o que apender e aprimorar, mesmo que o educador que deva direcionar este processo.

O enfermeiro tem um papel muito importante no cuidado ao estomizado, pois é ele quem oferece as primeiras orientações sobre a estomia, desta forma esclarece dúvidas, auxilia o paciente nos cuidados básicos, incentiva o paciente a melhorar a autoimagem (SOUZA et al., 2016).

Alguns pacientes transfere os cuidados de higiene e substituição da bolsa coletora a outra pessoa, geralmente o companheiro, devido a não aceitação da nova condição, dificuldade na visualização do estoma ou outro motivo (COQUEIRO; RODRIGUES; FIGUEIREDO, 2015). Os cuidados de saúde devem ser realizados abrangendo o paciente e seus familiares, pois estes geralmente estão completamente envolvidos nos cuidados e melhora a autoconfiança do paciente, além de melhorar a relação profissional-paciente-família (FREIRE et al., 2019).

O processo de educação em saúde também deve estar direcionado para a família, pois esta é essencial para a aceitação do paciente à nova condição de vida. A família está presente em todos as fases da doença, dando suporte à adaptação do paciente, sentido para lutar pela vida, oferecendo cuidados, proteção, conforto e afeto, mesmo que depois o indivíduo alcance a autoconfiança e autonomia. A família também possui dúvidas e angústias, e desta forma também devem ser acolhidos e apoiados (MENDONÇA ET AL., 2015).

O paciente colostomizado passa por mudanças em seu corpo que atinge sua autoestima, alimentação, vestuário, seu trabalho, suas relações sociais e sua sexualidade (MOTA; SILVA; GOMES, 2016). A nossa sociedade atual vive um mundo de aparências, com padrões de beleza e valorização do corpo, e a presença de uma estomia não se enquadra nos padrões sociais, acarretando dificuldades no indivíduo, especialmente no lado sexual. As alterações sexuais estão relacionadas a imagem corporal, afetando assim a autoestima e as relações do paciente com o parceiro, amigos e familiares. Desta forma, os estomizados necessitam recuperar sua sexualidade, e cabe aos profissionais possuírem conhecimentos e habilidades 
para abordar e oferecer suporte, de forma a ajudar nesse processo (VERA et al., 2017).

A estomização afeta a sexualidade do paciente, pois interfere nas suas relações sociais e afetivas. Diante disto, é exigido que o profissional de saúde intervenha nesta situação, de forma que entenda o viver do paciente e elabore estratégias que melhorem a autoimagem e autoestima, para que o paciente tenha uma vida sexual prazerosa e natural (MOTA; SILVA; GOMES, 2016).

Vera et al. (2017) apontam que sexualidade da pessoa estomizada é uma questão ainda pouco tratado pelos profissionais de saúde, de forma que a dificuldades de abordagem e questionamento sobre o tema. Seja a nível hospitalar ou ambulatorial, os profissionais omitem ou discutem de maneira superficial o assunto, devido o medo de causar constrangimentos ao paciente.

Muitas mulheres colostomizadas têm a sexualidade prejudicada devido obstáculos formados por elas mesmas, com vergonha da autoimagem e medo de rejeição. Desta forma, necessitam serem preparadas emocionalmente para reiniciarem sua sexualidade. Cabe ao enfermeiro elaborar intervenções educativas para que essas mulheres superem seus traumas, aceite e se adapte a colostomia para desfrutarem de uma sexualidade prazerosa. Para isso é necessário incluir os parceiros nas intervenções para eliminar medos e duvidas, e prepara-los para apoiar e ajuda-las a superar suas dificuldades natural (MOTA; SILVA; GOMES, 2016).

As alterações devido a estomia vão além do físico, mas englobam as emocionais, especialmente pela disfunção sexual devido a sentimento de culpa, rejeição, dificuldade de adquirir novos relacionamentos, vergonha, isolamento, medo de descolamento da bolsa de colostomia, de que o ato cause danos a mesma. Desta maneira, é uma fase de descoberta e adaptação (VERA et al., 2017).

\section{CONSIDERAÇÕES FINAIS}

Com base nas leituras apresentadas, ficou evidente que os cuidados de enfermagem ao paciente estomizado é voltado principalmente para as orientações 
sobre o dispositivo coletor. Muitos pacientes estomizado tem uma baixa autoestima e sentimentos de sofrimento medo, vergonha, tristeza, entre outros, e desta forma é essencial que eles recebam, informações adequadas, além escuta adequada e apoio psicológico e emocional.

É importante destacar que os cuidados de enfermagem ao paciente estomizado não pode estar direcionado apenas para a questão física do paciente e sua condição, mas observar o indivíduo como um todo, principalmente em seu lado psicológico, emocional, sexual e social. O enfermeiro deve oferecer um atendimento individualizado, incentivar a aceitação, adaptação, o autocuidado melhora da autoestima e autoimagem do paciente estomizado, visando a aceitação do novo estilo de vida, para que ocorra um convívio social satisfatório e assim o paciente tenha sua qualidade de vida melhorada. Além disso, o enfermeiro deve ter conhecimento e interesse suficiente para assistir a esta população. Assim, a assistência ao paciente estomizado deve ser melhor abordada durante a graduação e no ambiente de trabalho, pois nem sempre o enfermeiro será especialista na área, mas este deve estar apto a prestar orientações e informações adequadas ao cliente.

É importante ainda, o incentivo a pesquisas sobre o assunto, visto que houve dificuldades em encontrar obras direcionas especificamente aos cuidados do profissional de enfermagem ao paciente com estomia nos últimos cinco anos, mostrando que o tema ainda é pouco discutido no meio acadêmico e na área da pesquisa, devendo ser incentivado o estudo e a busca pela qualificação dos profissionais de enfermagem. 


\section{REFERÊNCIAS BIBLIOGRÁFICAS}

BARBURITTI, R. C. S, SILVA, M. C. P, e ABREU, M. A. L. Ostomia, uma difícil adaptação. Revista da Sociedade Brasileira de Psicologia Hospitalar, vol. 11, pag. 27-39. Disponível em: http://pepsic.bvsalud.org/pdf/rsbph/v11n2/v11n2a04.pdf. Acesso em: 30 nov. 2019.

BRASIL. Instrutivo de Ostomia: Portaria GM 793 de 24 de abril de 2012 e Portaria GM 835 de 25 de abril de 2012. Brasília: Ministério da Saúde, 2012.

Ministério da Saúde. GUIA DE ATENÇÃO À SAÚDE DA PESSOA COM ESTOMIA. Brasília: Ministério da Saúde, 2019. Disponível em: https://portalarquivos2.saude.gov.br/images/pdf/2019/julho/26/GUIA-ESTOMIA- ConsultaPubliaca-05-06-2019.pdf. Acesso em: 30 nov 2019.

Ministério Da Saúde. Instituto Nacional do Câncer (INCA). Estatísticas de câncer. Disponível em: https://www.inca.gov.br/numeros-de-cancer. Acesso em: 30 nov 2019.

Portaria № 400, de 16 de novembro 2009. Brasília: Ministério da Saúde, 2009. Disponível em: http://bvsms.saude.gov.br/bvs/saudelegis/sas/2009/prt0400_16_11_2009.html..Acesso em: 30 nov 2019.

COQUEIRO, J. M.; RODRIGUES, A. S. S. J. R.; FIGUEIREDO, T. A. M.; A produção do cuidado ao usuário estomizado: considerações da equipe de enfermagem. Revista de Enfermagem UFPE on line, Recife, v. 9, n. 6, p. 8148-54, 2015.

FREIRE, D. A. et al. Autoimagem e autocuidado na vivência de pacientes estomizados: o olhar da enfermagem. Revista Mineira de Enfermagem, 2017.

GEMELLI, L. M. G.; ZAGO, M. M. F. A interpretação do cuidado com o ostomizado na visão do enfermeiro: um estudo de caso. Revista Latino-Americana de Enfermagem, v. 10, n. 1, p. 3440, 2002.

MAURÍCIO, V. C. et al. A visão dos enfermeiros sobre as práticas educativas direcionadas as pessoas estomizadas. Escola Anna Nery, v. 21, n. 4, 2017.

MENDONÇA, S. N. et al. Orientações de enfermagem e implicações para a qualidade de vida de pessoas estomizadas. Revista de Enfermagem UFPE on line, Recife, p. 296-304, 2015.

MORAES, J. T.; SANTOS, C. F.; BORGES, E. L. Da formação à prática: a percepção de supervisores de enfermagem sobre os cuidados em estomias. Revista Enfermagem UERJ, Rio de Janeiro, v. 24, n. 2, 2016.

MOTA, M. S.; SILVA, C. D.; GOMES, G. C. Vida e sexualidade de mulheres estomizadas: subsídios à enfermagem. Revista de Enfermagem do Centro Oeste Mineiro, v. 6, n. 2, p. 2169-79, 2016.

NASCENTES, C. C. Rede social das pessoas com estomias: um subsídio para a atuação do enfermeiro. Tese (Mestrado em Enfermagem) - Rio de Janeiro: Escola de Enfermagem Anna Nery, Universidade Federal do Rio de Janeiro, 2015.

NIEVES, C. B. et al. Percepção de pacientes ostomizados sobre os cuidados de saúde recebidos. Revista Latina-Americano de Enfermagem, 2017.

PINTO, I. E. S. et al. Fatores de risco associados ao desenvolvimento de complicações do estoma de eliminação e da pele periestomal. Revista de Enfermagem Referência, n. 15, p. 155-66, 2017 
ROCHA, J. J. R. Estomas intestinais (ileostomia e colostomia) e anastomoses intestinais. Medicina, v. 44, n. 01, p. 51-6, 2011.

SILVA, N. M. et al. Aspectos psicológicos de pacientes estomizados intestinais: revisão integrativa. Revista Latina-Americano de Enfermagem, v. 25, 2017.

SOUZA, M. M. T. et al. Apoio emocional realizado por enfermeiro ao paciente ostomizado. Revista Portuguesa de Enfermagem de Saúde Mental, p. 49-56, 2016.

VERA, S. O. Sexualidade e qualidade de vida da pessoa estomizada: reflexões para o cuidado de enfermagem. ReOnFacema, v. 3, n. 4, p. 788-93, 2017. 